\title{
Concept and Formalisation of Tactile Cartographic Variables
}

\author{
Yi Rong Chen, Stephan van Gasselt* \\ Geomatics Group, Department of Land Economics, National Chengchi University, Taipei, Taiwan ((109257027;svg)@nccu.edu.tw). \\ * Corresponding author
}

Keywords: Tactile Maps, Vision Impairment, Visual Variables, 3D Printing

\begin{abstract}
:
With 2.2 billion people, vision impairment is a disability that affects approximately one third of the world's population $(W H O, 2019)$. The actual number remains unknown due to an imbalance of treatment cover in developing countries. The total numbers on severe vision impairment and blindness vary due to different legal frameworks, and national definitions. Despite generally improving medical services, the total number of vision impaired people will likely increase in the future due to the effects of overaging and lifestyles.
\end{abstract}

As our world is extremely dependent on visual input for stimulation and orientation, people with severe vision impairment rely on substitutes, commonly through audio and audio-tactile input. That substitution cannot work well in particular when it comes to communicating spatial information made for a conventionally visual communication medium through non-visual means. However, having access to usable maps would considerably improve the situation for the visually impaired as it is linked to "better spatial decision making, environmental knowledge, and independent travel" (Lobben \& Lawrence, 2012). To address this imbalance, various forms of cartographic products relying on tactile input have been developed over the past few decades in order to cover the specific needs from a visually impaired community (cf. Perkins, 2002; Rener, 2017; Wabiński et al., 2020). With lower cost and its high quality, 3D printing nowadays has become the most competitive technology in this field. Although the great number of preliminary efforts leap forward in tactile mapping, it has been argued that progress made by sighted people is likely more technology-centric rather than human-oriented (Perkins, 2002), which probably reflects the situation in academia.

Here, we follow-up on existing research discussing tactile cartography for vision impaired people that is based on communication with members of the community and which tries to establish a workable concept rooted in experience and interaction with the community (e.g., Lobben \& Lawrence, 2012). Our research attempts to establish a conceptual cartographic foundation in which the technology is embedded as a tool to express rather than allowing the technology to govern the conceptual aspects. In that respect we discuss (1) a first-order conceptual model which includes the foundation of the cartographic model including syntactic and semantic aspects and translation of semantics into accessible pieces of information for the community; (2) a translation using an abstract and thus technology-independent formalization independent of the actual physical device (logical model), and (3) an actual implementation using the technology that is available and affordable to accomplish this approach, i.e., the physical model.

The conceptual design attempts to tackle the issue of formalization of tactile variables based on the existing concepts for graphic (visual) variables as communicated by Bertin (2010) and following researchers in the field of cartography and communication. Here, the cartographic idea is originally transferred through its concept into a representation by making use of 2D visual variables. Our approach covers the translation of 2D geometries and visual variables into 3D tactile map variables enriched by semantic information. One of our aims is to establish a conceptual and logical model that allows us to create such products from existing spatial map information. However, we also feel that this concept must be able to cope with a higher information complexity, i.e. maps that are not only made for navigation purposes, but also for education of, e.g., political, economic and social aspects. Our secondary aim in this research attempts to explore these limits by establishing a conceptual model which builds on (a) primary visual communication theory, and (b) lessons learned from existing standards and research results (e.g., Lobben \& Lawrence, 2012; Perkins, 2002; Bertin, 2010; Amick \& Corcoran, 1997; Edman, 1992).

In its first step, the core of this research and development project was to find a way to translate visual cartographic variables as proposed by Bertin (2010) and later work, into a complete set of tactile cartographic variables and investigate their usability, including limits of perception, within the vision impaired community. Limits of 
differentiability of tactile features, such as size, texture, orientation and shape properties are one aspect, the other one is the perception of the "larger picture" and the way how tactile variables become integrated into the spatial mental model with their selective or associative properties. Yet another aspect of the conceptual model is the translation of usually well-defined spatial $\mathrm{xy}(\mathrm{z})$ geometries into a 3D model by reducing geometric complexity to the minimum without losing information about the general settings of the map topic. The aim is not to create a naturalistic physical 3D model of the real world, but to abstract this reality into a model that is topologically accurate, and geometrically as accurate as needed in order to leave room for hosting additional semantic information. The purpose of this approach is to generate a map that is able to communicate selective properties despite being non-visual in nature.

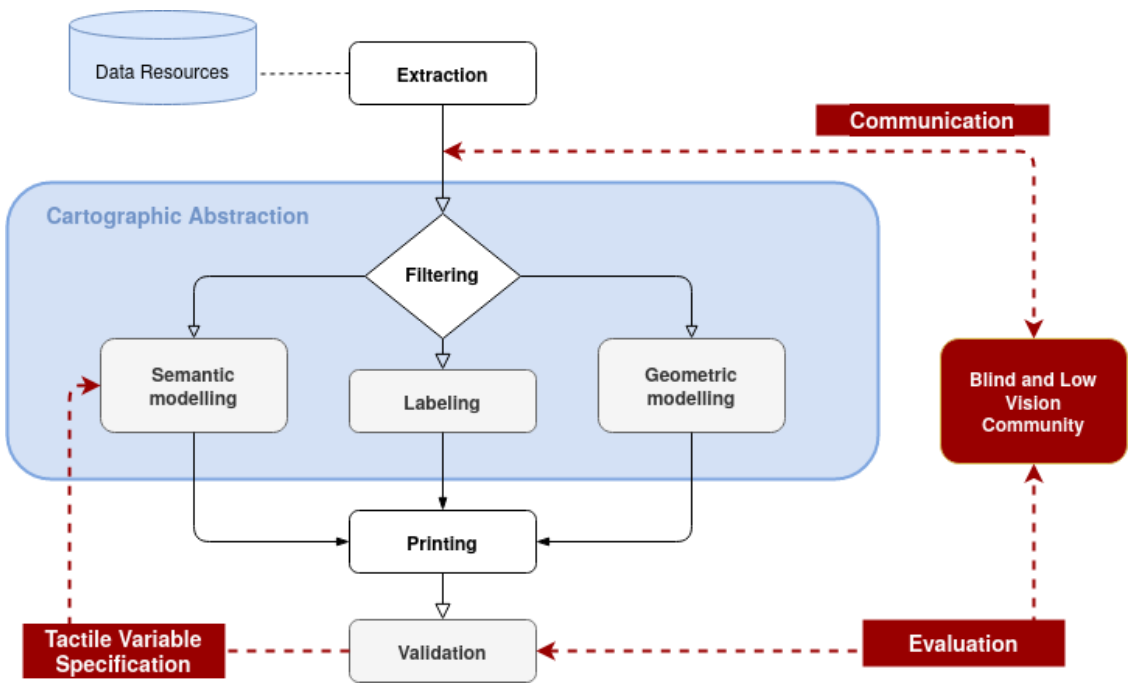

Figure 1. Workflow for establishing a conceptual model for development and community communication.

The development of conceptual models relies on experience and community feedback. The initial physical model implementation is required to allow testing and to facilitate communication within the community. During the current development stage, geometry is extracted through readily accessible APIs for the Open Street Map service. Geometry and semantic information are filtered and translated into 3D features, including simplification and generalization using the programmable OpenSCAD environment including library extension. For testing and prototyping we have decided to use Liquid Crystal Display (LCD) technology due to its higher resolution required for tactile feedback. At this stage we have finished building a first conceptual model of the use of tactile variables as part of the cartographic abstraction in Figure 1. We are currently working on feasible printing approaches to be able to perceive different qualities and to investigate tactile and technical resolution limits by taking into account common community guidelines (e.g., Amick \& Corcoran, 1997; Edman, 1992). The conceptual model will be part of the presentation during the conference.

\section{References}

WHO (2019): World report on vision. - xvi+162pp., World Health Organization, Geneva, Switzerland (WHO).

Lobben, A. \& M. Lawrence (2012): The use of environmental features on tactile maps by navigators who are blind.The Professional Geographer, 64(1), 95-108.

Perkins, C (2002): Cartography: progress in tactile mapping.- Progress in Human Geography 26,4: 521-530.

Rener, R. (2017): The 3D Printing of Tactile Maps for Persons with Visual Impairment - In: Antona, M. and Stephanidis, C. (Eds.) UAHCI 2017, Part II, LNCS 10278, 335-350, Springer, Heidelberg.

Wabiński, J., Mościcka, A., Kuźma, M. (2020): The Information Value of Tactile Maps: A Comparison of Maps Printed with the Use of Different Techniques. - The Cartographic Journal, DOI:10.1080/00087041.2020.1721765.

Bertin, J. (2010): Semiology of graphics: diagrams, networks, maps (translated from Sémiologie graphique, 1967). Redlands: ESRI Press.

Amick, N., Corcoran, J. (1997): Guidelines for Design of Tactile Graphics. American Printing House for the Blind, http://faculty.sfasu.edu/cadyd/indexpagextra/Science.Math/tactile.graphics.docx (accessed December 202020 ).

Edman, P. K. (1992): Tactile Graphics.- American Foundation for the Blind, New York. 